\title{
Entrevista a Flavia Terigi
}

\author{
Victoria Baraldi
}

Flavia Terigi es licenciada en Ciencias de la Educación, magister en Ciencias Sociales, especialista en Políticas Educativas y doctora en Psicología. Docente regular de la Universidad Nacional de General Sarmiento y de la Universidad de Buenos Aires. Es autora de numerosos libros y artículos científicos y de divulgación sobre temas de aprendizaje, curriculum y formación docente. El 9 de agosto de 2017 desarrolló la Conferencia «Trayectorias educativas y educación rural» en la Facultad de Humanidades y Ciencias de la Universidad Nacional del Litoral (FHUC-UNL). En el transcurso de la visita a nuestra Facultad tuvimos la posibilidad de realizarle esta entrevista.

Desde hace muchos años, a través de distintas publicaciones y seminarios, pusiste en tela de juicio la idea de «cronosistema escolar» y trabajaste con categorías específicas para procurar mirar las prácticas y el tiempo escolar desde otras perspectivas. Enfatizaste la distinción entre «trayectoria teórica» $y$ «trayectoria real», la idea de "cronologías de aprendizaje», discutiendo también otro de los principios como el de la «simultaneidad». Hiciste docencia —a través de cursos, conferencias, dirección de tesis - desde estas perspectivas para mirar los procesos de enseñanza y de aprendizaje. 
Las preguntas son: ¿qué cosas se han logrado conmover a partir de mirar de otra manera algo que se miraba de un modo tan estandarizado? ¿Podés registrar desde la docencia, desde la dirección de las escuelas, desde la investigación, otros modos de mirar los procesos escolares? Más allá de las malas interpretaciones que luego se hicieron del concepto de "trayectorias escolares», como mencionaste en la conferencia de hoy, ipodés registrar otras maneras de investigar, otras maneras de intervenir desde la docencia, desde la dirección de escuelas? En definitiva, ¿qué es lo que se ha podido conmover?

Muy buena pregunta. En realidad, yo trato al concepto, la idea de las trayectorias, como una perspectiva a la que le pasa algo de eso que hoy llamábamos "lo estelar». Esto ocurre cuando un concepto o una perspectiva en algún momento dado se hace muy visible, muy valorado, muy difundido y después se va incorporando al modo más estándar de pensar. Por eso yo terminaba la reflexión de hoy diciendo que en ciertos discursos uno reconoce que el concepto viene a ocupar de manera eufemística el lugar que antiguamente ocupaban las dificultades de aprendizaje.

Quizás una buena pregunta sería: ¿por qué se estelarizó? El porqué se hibrida, ya lo sabemos. Pero, ¿por qué se estelarizó? Porque al principio cuando nosotros lo empezamos a trabajar $-\mathrm{y}$ no fuimos los únicos - no nos imaginábamos que iba a tener la repercusión que tuvo.

A mí me parece que el concepto de «trayectoria», la perspectiva en general, contribuye a comprender un poco mejor procesos escolares en donde intervienen (de forma combinada en la producción de efectos) algunas condiciones que son estructurales, y algunas condiciones que son del sujeto. Ese enlace que logra hacer la perspectiva yo creo que fue potente para romper algunas formas de mirar lo educativo que no estaban funcionando. Es decir, explicarlo todo por lo estructural o explicarlo todo por el sujeto. Esto no condecía demasiado con la vivencia de muchos de los profesores, profesoras, maestros y maestras que se daban cuenta de que "ahí hay algo más», y que ven en las propias condiciones del sistema escolar un límite para poder ensayar otras cosas.

Tal vez, un efecto que se puede reconocer (que no es universal, simplemente que yo lo percibo), es una mayor capacidad de muchas personas para tratar de conectar lo que se presenta como dificultad de aprendizaje, rezago escolar, abandono, deserción, repitencia... con condiciones de sistema. Yo creo que hay más capacidad para pensar eso en el ámbito específicamente educativo. Afuera de la escuela, fuera del ámbito más especializado de las personas que trabajamos en el mundo de la escuela, no creo que haya tenido mucho efecto. En verdad, se convierte en una especie de emblema o de lema que, como tantos otros lemas, se le hace decir cosas que no dijo o se lo utiliza para cuestiones para las cuales no fue generado. $Y$ tiene su lógica, porque al final no deja de ser una perspectiva un poco hermética para quien no viene del campo educativo.

Yo considero que el concepto sirvió para entender mejor las relaciones entre condiciones del sistema y las cosas que les pasan a los pibes. Es una perspectiva que tiene dos modos de abordaje principales. Una es trabajar a escala de poblaciones y entonces se trabaja con información estadística. Yo creo que ha tenido un fuerte impacto en aprender a mirar la estadística de otro modo. Hemos oído hablar de la estadística de muchas maneras, y eso es muy bueno porque sino la estadística es la parte árida de lo educativo, la que siempre «tengo que poner como fundamentación del problema» pero después no interviene en el problema como tal. Yo creo que se 
generaron otros modos de mirar la información estadística y se reconocen límites que tiene para poder advertir ciertas cosas; esa es una vía.

La otra vía es la de la entrevista, del enfoque más biográfico. Creo que por esta otra se produjo mucho material que ha sido muy valioso: entrevistas, relevamientos, historias de vida de los chicos, documentación de situaciones que los sujetos relatan en sus entrevistas con nosotros y con otros equipos de investigación. Queda un material muy rico para comprender la perspectiva del sujeto, de quitarles a los adolescentes esto de "no les interesa», "no se ponen las pilas», "todo les da lo mismo». Mostrar el sufrimiento del sujeto a través de algunas de las situaciones que la estadística describe como repitencia, abandono, creo que pudo haber sido un aporte. No creo que se haya incorporado todavía de manera fuerte como perspectiva conceptual, tal vez falta producción. Hay muchas tesis y demás, pero falta algún género más adecuado, como puede ser el artículo o inclusive el libro, ya que todavía es una producción un poco pesada. Recién me pedían mi tesis a mí, piden la tesis de Bárbara Briscioli... ${ }^{(1)}$

(1) Se refiere a su tesis doctoral que no está publicada, y a otra tesis que ella ha dirigido incorporando esta perspectiva.

\section{¡Ahí tenés para publicar!}

Es que el problema no es que nosotros no publiquemos, sino que estamos publicando en un género que se difunde mucho, que es la publicación online, y esto también explica la difusión. Yo tengo una conferencia y la cuelgo, un artículo y no lo publico en una revista, lo pongo a disposición. No le hace muy bien a mi carrera académica pero contribuye mucho a la difusión del tema, lo torna muy accesible, y eso está bueno. Pero faltaría un material intermedio que permita incorporarlo en un programa, algo más estandarizado como lo que quiere el sistema.

En términos de intervenciones, yo creo que la perspectiva de las trayectorias llenó un poquito el discurso educativo del gobierno, sobre todo el del primer gobierno de Cristina Kirchner. Hablo en términos educativos, de la política educativa. Y esto no necesariamente significa que haya tenido implicancias en la definición de la política. Yo todavía hoy me lamento y me pregunto por qué no se tomó una decisión más estructural respecto de la escuela secundaria. Está todo a la vista, no es que no sabemos lo que pasa o parte de lo que habría que hacer, pero no se tomó una decisión cuando había buenas condiciones para tomarla y ahora son unas condiciones mucho más difíciles.

Estamos en un contexto de mucha simplificación del problema educativo, de esgrimir soluciones simples para problemas que son muy complicados, de mucho desconocimiento de lo que ya se hizo, de decir alegremente cosas que ya se probaron y que no funcionan. Entonces, esta no es una buena coyuntura para pensar que van a pasar otras cosas en la escuela media. Yo no creo que haya habido tanto impacto político educativo, sí hubo impacto de experiencias. Podés encontrar proyectos escolares, 
algunas experiencias más pequeñas en las cuales sí hay atención a la problemática de las trayectorias de los sujetos y se buscan modos de acompañar con cambios institucionales lo que ya sabemos que es un descoloque entre los sujetos y el sistema.

$Y$ en el marco de esta primera pregunta, iquisieras comentarnos brevemente la experiencia de la escuela que estás asesorando, que es una escuela secundaria que depende de la Universidad Nacional de General Sarmiento?

Es una escuela que diseñamos a lo largo del año 2014 y comenzó a funcionar en el año 2015 en el marco de un proyecto que tenía por entonces el Ministerio de Educación de creación de escuelas secundarias en universidades nacionales. Yo creo que se generó porque las universidades eran el terreno en el cual el Ministerio de Educación Nacional tenía oportunidad de crear escuelas interviniendo más directamente en el proceso. Por esto, se abrieron tres escuelas en 2014 , dos más en 2015 y alguna más que se sumó en el 2016.

El proyecto ministerial tenía, a mi criterio, el propósito de generar otros modos de pensar la escuela secundaria. Nosotros, en la Universidad Nacional de General Sarmiento, somos una universidad con mucho recorrido en el tema de educación secundaria, y teníamos mucho material acumulado. Tomamos la propuesta del Ministerio, decidimos no abrirla en el primer año, y dedicar todo 2014 a discutir el modelo que nos trajeron y armar uno más adecuado. En ese sentido, de todas las escuelas secundarias que se crearon, la nuestra es la única que tomó una hipótesis totalmente distinta a la ministerial, que era la de incrementar el tiempo de los chicos en la escuela, de pensar una escuela secundaria más contenedora, pero sin tocar el régimen académico.
Nosotros decidimos hacer del régimen académico la principal línea de intervención. De este modo, generamos una escuela secundaria que tiene un régimen académico muy diferente al de la escuela secundaria común en el que se promueve el cursado por materias. Cada estudiante tiene definido en cada periodo escolar un trayecto académico propio en el que combina las materias que está cursando, las actividades de apoyo en las que participa, la formación opcional por la que va inclinándose. Por ejemplo, aunque tenga que hacer un recorrido por todos los lenguajes artísticos a lo largo de su escolaridad, puede decidir por cuáles empieza y cómo va circulando y muchas actividades de formación ocasionales, complementarias que se van sumando al recorrido.

La principal innovación de la escuela va por este lado del régimen académico. Un régimen de estas características promueve una revolución en la organización escolar y a los profesores se les presentan muchos desafíos. Por ejemplo: desarrollar para un grupo escolar más de una «cronología de aprendizaje», proponer una mirada muy atenta a cada sujeto como para no retenerlo en un nivel de aprendizaje que ya podría estar promovido al nivel que le sigue, genera una dinámica cotidiana muy complicada y cierta obligación de producción de conocimiento. En eso estamos...

\section{¿Y se están viendo respuestas positivas por parte de una idea de pertenencia a la escuela? ¿Los chicos quieren que- darse? ¿Se generan cambios cognitivos importantes?}

Tenemos en primer lugar una retención altísima. Nosotros mismos no pensábamos que iba a tener el efecto que efectivamente está teniendo en términos de permanencia de los chicos en la escuela. Eso es bastante impresionante. 
En cuanto a la pertenencia a la escuela, nosotros nos preguntábamos si el hecho de que los chicos variaran de grupo no iba a generar alguna clase de dificultad a la hora de crear lazos adentro de la escuela. Se van armando grupos más o menos estables. Sucede como en la universidad, compartís con algunos compañeros cuatro o cinco materias, otras las hacés por tu cuenta y conocés otra gente. La verdad que lo que produce esto es que los compañeros de un chico son todos los de la escuela. Esto genera una situación social menos enclaustrada que lo que te ocurre cuando pertenecés a un único grupo donde muy rápidamente se tipifican los roles y demás. Te diría que ese es un aspecto que no habíamos buscado especialmente pero que se hizo interesante. Aumenta la sociabilidad de los chicos en función de que están con muchos más compañeros.

\section{Más dinamismo también, porque a veces los cursos se fragmentan en dos grupos y quedan los cinco años así frag- mentados.}

Tal cual, acá los chicos van variando, no es que no intenten quedarse con sus compañeros haciendo las mismas cosas pero el propio régimen los lleva a cierta movilidad.

En lo que tiene que ver con los procesos de aprendizaje de los chicos, lo primero que hay que advertir es que nosotros trabajamos en una zona de la provincia de Buenos Aires de una cierta vulnerabilidad social. Los estudiantes vienen de recorridos en la escuela primaria que los colocan de maneras muy desiguales en el inicio de la experiencia de educación secundaria. Es impresionante la diversidad de aprendizajes con los que llegan. Es decir, nada que un profesor o profesora de una escuela secundaria común no se pueda imaginar, pero rompe un poquito la figura del ingresante a la escuela de la universidad.

Nosotros trabajamos con la población real del barrio, porque para poder ser alumno de la escuela el único requisito es vivir en un cierto radio de la escuela y haber obtenido la vacante por sorteo. Y una parte de nuestro aprendizaje ha tenido que ver con empezar a tener una propuesta educativa más adecuada a los aprendizajes previos de los chicos. Nosotros mismos no estábamos tan preparados para la situación inicial con la que los chicos se presentaron. $Y$ todavía estamos tratando de definir algo — que puede parecer simple pero que exige acordar con los profesores- que es: ¿qué esperamos del ciclo básico? ¿Cuáles son los aprendizajes que nosotros esperaríamos que los chicos pudieran lograr en el ciclo básico y que no son de una única disciplina? ¿Qué le entrega el ciclo básico al superior? Con relación a la pregunta sobre qué aprendizajes promovió el ciclo básico, tenemos algunos acuerdos. Por ejemplo, los chicos tienen que haber aprendido: estrategias de aprendizaje colaborativo y a interpretar y representar la información en diferentes formatos. En fin, tenemos un pequeño listado de objetivos de aprendizaje y que vamos cuidando que se promuevan en las diferentes asignaturas.

En cuanto a la secuencia de contenidos más clásica, nos está costando un poco encontrarle una definición igual de cómoda a qué debería ser el ciclo básico en Matemática, en Lengua o en Historia. Por un lado, los profesores que tenemos en la escuela son profesores que han sido formados — como todos los profesores - para desarrollar currículos muy extensos con muchos contenidos. Nos cuesta promover y lograr que ellos acepten seleccionar algunos núcleos de contenidos y trabajar intensa- 
mente con ellos. Lo logramos con algunos, otros lo saben hacer porque ya lo venían ensayando pero hay profesores que todavía no se atreven a sacrificar un poco de la extensión en mérito de la profundidad. Ese es el dilema en secundaria: la extensión del campo de una disciplina y la decisión que tiene que tomar el profesor de seleccionar e ir en profundidad con algunas estructuras conceptuales, eso cuesta mucho.

Que es un desafío que está siempre presente, pero quizá se haga más difícil de resolver en estas instancias.

Y claro, porque en la escuela secundaria común el nivel uno de matemática te lo dicta el año escolar. Pero acá vos podrías tener un grupo donde una parte de los chicos está en el nivel uno de matemática y una parte está en el nivel dos pero hay puntos en lo que todavía tenés que definir qué es exactamente el nivel...

Hay campos que lo tienen más desarrollado, como las lenguas extranjeras. La gente que enseña lenguas extranjeras y que tiene una formación medianamente actualizada, entiende que de las cuatro grandes competencias que las lenguas tienen que formar, la formación es asincrónica. Entonces no es extraño que las capacidades de lectura estén más desarrolladas que las de escritura, y eso se ve como algo propio del aprendizaje de una lengua. Eso te pasa en cualquier asignatura, sin embargo, los profesores de otras disciplinas - que no tienen un desarrollo didáctico tan atento a este tipo de asincronías - piensan de manera muy sincrónica. Las cosas tienen que ir al mismo tiempo, tienen que ocurrir todas juntas. Por lo tanto, cuesta determinar cuáles serían las asincronías aceptables para un cierto nivel de aprendizaje de una materia.
$Y$ además requiere, me parece, de un mayor conocimiento de la propia disciplina para tomar esas decisiones, de cuáles son las estructuras sintácticas, las estructuras sustantivas, lo propio de la generación de conocimiento en ese campo disciplinar, para luego definir qué es lo que se tiene que enseñar.

Absolutamente, y requiere una mirada del contenido. Y esto es un problema de la formación general de los profesores de secundaria, se requiere una mirada del contenido que no es la de la estructura de la disciplina, es la mirada del aprendizaje y por lo tanto de la enseñanza, y está muy poco desarrollada. En la formación de los profesores todo el circuito pedagógico didáctico está muy disociado del trabajo sobre los contenidos...

Sí, claro, porque el docente a veces se forma como el especialista en una disciplina por un lado y no se articula con la formación pedagógica. Entonces, la pregunta retórica y muy antigua: iqué es lo necesario de esta disciplina, para este sujeto, en esta circunstancia? Si no estás formando a un especialista, la pregunta es: ¿qué le hace falta a este sujeto para vivir hoy y proyectarse en el futuro?

Y para seguir aprendiendo dentro del mismo campo, digamos. Yo siempre pongo el ejemplo de matemática, porque es muy claro. Todos los que hemos egresado de la escuela secundaria nos hemos pasado cinco horas de nuestra semana durante doce años de nuestra vida aprendiendo matemática. $Y$ la mayor parte de la gente solo conoce a fondo sistema de numeración, las cuatro operaciones elementales, alguna cosa de proporcionalidad y no mucho más... Algo está mal. 
(2) Escuelas de Familia

Agrícola.

Creo que es porque se enseñó una versión distorsionada de la matemática. Se enseñaron rituales matemáticos, probablemente...

Rituales en los que alguno enganchó y siguió la cronología y entonces...

Sí, es todo un tema. Ahora hay equipos de docentes investigadores que están revisando muy profundamente la enseñanza de la matemática. Lo que se genera en la autoestima cuando no se puede aprender, y el sentido social que hoy puede tener la matemática...

Sí, crear en los chicos una posición de dominio del conocimiento matemático porque con la situación de que no entendés nada...

Nada, ni por qué lo aprendés, ni por qué te lo enseñan, para qué lo querés... Eso es paralizante. Yo creo que hoy ningún adulto aprendería algo que no sabe el por qué ni para qué lo aprende. Sin embargo a los chicos constantemente se les exige que aprendan cosas que no saben para qué las aprenden. Entonces, es un mecanismo de resistencia. Para qué invertir tiempo y energía en algo que no sé para qué es.... Tal cual...
Pasando a otro tema, quisiera preguntarte algo más, a propósito de tu especialización en educación rural. Yo te he escuchado decir, y comparto, que hay poca sistematización pedagógica, estadística y de experiencias sobre educación rural. Hay algunas excepciones como algunos casos de maestros emblemáticos, 0 las EFA ${ }^{(2)}$ que acá en el litoral han sido muy importantes. Hoy hay un cambio en la conformación de lo rural, porque también hay cambios en los modos de producción y otros dados por el acceso a internet. La pregunta es: icómo estás viendo la educación rural? ¿Cuáles serían las particularidades de la educación rural hoy? Bueno, lo primero, como vos bien sabés, el problema que quiero señalar es que la categoría de «educación rural» en verdad es más una categoría residual de lo urbano y entonces es una categoría que encubre una diversidad de situaciones muy importantes. Para un docente urbano, rural es todo lo que no sea la educación que conoce. Y ahí está la situación de la ruralidad como vos planteás donde está cambiando el modelo productivo y entonces, por ejemplo, se despuebla el campo, y escuelas que antiguamente tenían tamaño pasan a ser escuelas muy pequeñas. Hay otras situaciones, por ejemplo, la ruralidad en el circuito mapuche en el sur. Es otra cosa completamente diferente, porque ahí funciona la escuela dentro de un enclave más comunitario. La dinámica poblacional es muy distinta y la relación con la cultura oficial transmitida por la escuela también es muy diferente. Entonces, en ese sentido, primero destacar lo diverso de lo rural como también es diverso lo urbano. Pero lo rural está invisibilizado en esa diversidad. Creo que si le pidieras a la gente que dibujara una escuela rural, dibujaría una escuela de campo, chiquitita. No se imagina las EFA, ni tampoco la escuela de la comunidad mapuche, no se imagina modos escolares que son muy significativos en el campo rural. 
Lo segundo es que lo que nosotros tomamos de lo rural, quizás no sea lo más interesante de lo rural pero sí es lo que le es específico en términos de organización escolar, que es la escuela rural pequeña. En ese sentido, no tengo mucho para decir sobre escuelas rurales de mayor tamaño, porque lo que a nosotros nos interesó originalmente de la escuela rural es que la imaginábamos en razón de la imposibilidad de tener un maestro por grado. La imaginábamos como una especie de interesante laboratorio para que pasen otras cosas en términos didácticos algo así como: «si hay un lugar donde tenés que romper el monogrado es en la escuela rural pequeña».

Entonces empezamos a estudiar la escuela rural por ese motivo. $Y$ nos encontramos en nuestros primeros estudios con que -lejos del laboratorio que te permite imaginar algún maestro emblemático como Iglesias o Gesualdo en Uruguay - algunos maestros lograban la ruptura con la lógica de la escolaridad graduada que traían aprendidas de su formación. Pero la mayor parte de las situaciones que encontrábamos eran situaciones en las que los maestros intentaban reproducir en el contexto del plurigrado el modelo escolar de "cada chico tiene que estar con cosas propias de su grado" y eso llevaba $-\mathrm{y}$ lleva- a segmentar la jornada escolar de manera muy curiosa. Por ejemplo, organizar el espacio para que estén bien diferenciados cada uno de los grados escolares y disminuir mucho el tiempo de trabajo de los maestros con cada grupo. Porque, además, la formación docente no te prepara para habilitar a los chicos a trabajar autónomamente. Entonces hay herramientas de enseñanza que los maestros no tienen para poder hacer este tipo de trabajo.
Recuerdo una escena que para mí es de esas que son paradigmáticas del problema que estamos conversando. Ocurrió en una escuela rural pequeña: una maestra tenía un grado imposible con treinta y un alumnos. Al año siguiente ya contaba con dos maestros, pero ese año tenía solo una con esa cantidad de chicos, de los cuales dieciséis estaban en primer grado y pertenecían básicamente a tres familias de la comunidad. Antes no había escuelas, entonces cuando esta se abrió las familias mandaron a sus hijos. Entonces había, por ejemplo, de una misma familia un chico de seis y todos sus hermanos, hasta la mayor de quince. La escuela tenía alumnos en grados superiores -quinto, sexto y séptimo- que se habían cambiado a esta escuela porque les quedaba más cerca.

De este modo, se producían situaciones donde de pronto los chicos de los grados superiores estaban entrando, por ejemplo, a aprender un modo de representación con el que nunca habían trabajado (como los mapas), y la maestra no podía pensar que a la de quince - que tenía en primer grado- con relación a los mapas, podía perfectamente ponerla a trabajar con los chicos más grandes. ¿Por qué la maestra no puede pensar eso? Bueno, porque su mentalidad está constituida por la escuela graduada y comparte la idea de que si todavía no sabe leer y escribir, los otros modos de representación no los puede aprender. Ejemplo muy claro de algo que no es responsabilidad de la maestra, es cómo ha sido formada. Bueno, nosotros pensábamos que íbamos a encontrar allí una especie de laboratorio que no encontramos y entonces a partir de allí nos pusimos a 
estudiar mucho. Primero, cómo ocurre la enseñanza en los plurigrados, qué problemas se les presentan a los maestros que ellos dicen que no pueden resolver y cómo resuelven algunos de ellos. Después ya en la etapa más abocada a la investigación empezamos con algunos maestros, con algunas escuelas a trabajar intentando promover algún tipo de actividad que propiciara el trabajo con un conjunto de chicos, de nenes y de nenas que estuvieran en distintos grados de la escuela rural. He trabajado mucho en Paraná con escuelas rurales del departamento, en cuestiones de aprendizaje del sistema de numeración del primer ciclo, promoviendo una experiencia de trabajo conjunto con chicos de grados diferentes con la UADER ${ }^{(3)}$ en particular con la Escuela Almafuerte.

(3) Universidad Autónoma de Entre Ríos.

\section{...Que fue la primera escuela rural americana.}

Exactamente, para nosotros fue genial vincularnos con la Escuela Normal Rural Almafuerte. Es un lugar simbólico. Nos relacionamos con los profesores de Práctica, de Residencia, con la profesora de Didáctica de Matemática, y con los residentes para generar una propuesta didáctica. Fue interesante y ahora volvimos a trabajar allá en la zona de Buenos Aires, con escuelas de San Andrés de Giles. Entonces, iqué destacaría de todo lo que se puede decir de lo rural? A mí lo que me interesa es la singularidad del modelo organizacional cuando la escuela rural pequeña pone un docente al comando de un grupo de chicos que está en distintos grados escolares.

La cuestión de las tecnologías que me preguntabas es particularmente interesante pensando en la educación secundaria. Siempre el problema es que necesitás al especialista —que es el profesor-, pero no tenés la posibilidad de que efectivamente se haga presente con la asiduidad con que te haría falta en el contexto rural. Entonces pienso que ahí hay una punta para comenzar, hay algunos proyectos nacionales al respecto, hay otros países que tienen mucho desarrollo sobre esto. Pienso que por ahí puede venir alguna alternativa que combine presencialidad con apoyos utilizando tecnologías, pero no es un punto en el cual yo me esté deteniendo particularmente. Por ahora, en las escuelas primarias donde yo trabajo, el equipamiento es muy insuficiente, la conectividad es bajísima. La escuela primaria rural si tiene computadora tiene el modelo del aula laboratorio y ese no nos sirve. Porque no es que los chicos tienen que ir a hacer unas horas de computación, los chicos tienen que tener incorporadas 
las herramientas para el trabajo en el aula. $Y$ para eso, tenés que tener buenas computadoras y buena conectividad. Los maestros pueden usar un pen drive pero es como sacarle fotocopias a la enciclopedia. No es encontrar la potencia que tiene la conexión online, la búsqueda de información, el trabajo con herramientas que permiten construir simulaciones, todo eso está muy lejos todavía, me parece a mí...

0 sea que la tecnología no está modificando los modos de enseñar en términos generales...

Las escuelas que yo conozco son escuelas que piensan que los chicos tienen que conocer las computadoras, pero no están pudiendo incorporar las computadoras al trabajo en el aula. Los chicos saben lo que es una computadora, en general todavía no tienen celular, pero saben lo que es. $Y$ el celular probablemente a futuro sea mucho más dúctil que la computadora para hacer ciertas cosas en el aprendizaje. Pero no hay mucho más que eso. No hay buenas condiciones de conectividad y te estoy hablando de la provincia de Buenos Aires, a cien kilómetros de la capital, no te hablo de un paraje pequeño; te hablo de San Andrés de Giles, pleno corazón de la producción sojera. Mucha riqueza en un montón de cosas pero no en la conectividad a Internet.
El eje de esta revista es «jóvenes y educación». Nos pareció muy importante entrevistarte, porque más allá de los desarrollos teóricos que realizaste, por debajo hay una preocupación genuina por los jóvenes y la educación. Como esta es una revista dirigida en particular a docentes, iquerés agregar algo más dirigido a ellos?

Sí, claro. De todo (risas). Primera cuestión, la perspectiva con la que nosotros trabajamos se conoce genéricamente como la de las «trayectorias escolares». Esta perspectiva aporta mucho para entender la configuración que se arma entre la escuela y la vida de los sujetos, y aunque uno no haya leído mucho de la perspectiva, construir esa sensibilidad es extremadamente importante. A mí me parece que los profesores de secundaria tienen que ubicarse todo lo posible como profesores de adolescentes, de jóvenes, tan fuertemente como se definen en tanto profesores de Historia o de lo que sea. Es decir, la identidad profesional ojalá se pudiese construir no solo en torno a la disciplina sino también en torno a quien se dirige, a quien vos querés apasionar con tu disciplina.

El segundo aspecto es que a la escuela secundaria en Argentina, como en otros lugares del mundo, un poco le pasa esto de haber surgido como una escuela justificada para otro nivel educativo, una escuela para la universidad, una escuela para el trabajo. $Y$ yo quisiera que fuera una escuela para los adolescentes, para los jóvenes, una escuela que tenga sentido no solamente para el futuro sino para el presente de la vida de los chicos.

La experiencia que yo puedo transmitir es que cuando la escuela se convierte en un lugar donde transitar el proceso adolescente, que es un proceso muy complicado en nuestras sociedades, los pibes responden a la escuela. Responden como los pibes que son, se van a mandar todo tipo de «macanas» 
porque forma parte de andar probando, incluso de no saber dónde terminan algunas cosas que pueden hacer y algunas cosas que no, pero la escuela es un lugar muy importante en la vida de los adolescentes. Los profesores a veces piensan que los chicos no quieren estar en la escuela. Los chicos quieren estar en la escuela. A veces, lo que no quieren es estar en clases, cuando la clase toma esta forma de transmisión desvinculada de la contemporaneidad o desvinculada de la pasión que te puede generar un cierto conocimiento que no tenga aplicación directa con el presente.

Me parece que hay que ayudar a los profesores a verse a sí mismos como profesores de adolescentes, de jóvenes. Pasa que para los adultos, los adolescentes, los jóvenes son una categoría indivisa. Todos hemos aprendido a decir que hay «muchas infancias», «muchas adolescencias», «muchas juventudes» pero en el fondo hay una visión estereotipada: «son muchos todos juntos». Además me parece que, sobre todo para los que tenemos más edad, la adolescencia y la juventud, pero sobre todo la adolescencia de los chicos de hoy en día es muy distinta de lo que fue la nuestra. Entonces hay una dificultad para reconocerse uno mismo ahí, en un adolescente que vive cosas muy diferentes de las que podemos haber vivido nosotros. Algo de romper la diferencia intergeneracional, no desconociéndola, pero primero desetiquetando. Los adolescentes son muy diversos sujetos, con muy diferentes circunstancias, y comprender que están viviendo un mundo muy distinto del que vivíamos nosotros cuando teníamos su edad, y que si a nosotros nos desconciertan algunas cosas de este mundo, imaginate a ellos. Que dominen ciertas cosas con mucha rapidez, como puede ser, por ejemplo, la tecnología, no los hace sujetos preparados para entender algunas cosas del mundo contemporáneo. $Y$ nosotros podemos tener ahí un lugar interesante de construcción, de comprensión, de acompañamiento, de ayudarlos a descubrir intereses que no tienen por qué tener si nadie les muestra algo por lo que interesarse. Hay algo de crianza en la escuela secundaria y me parece que es algo que yo les pediría a los colegas que trabajan en ese nivel educativo.

\section{Bueno Flavia, un gusto.}

Igualmente. 